\title{
Clinical implications of dihydropyrimidine dehydrogenase expression in patients with pancreatic cancer who undergo curative resection with $S-1$ adjuvant chemotherapy
}

\author{
MASAAKI MURAKAWA $^{1 *}$, TORU AOYAMA $^{1 *}$, YOHEI MIYAGI ${ }^{2}$, YOSUKE ATSUMI $^{1}$, \\ KEISUKE KAZAMA $^{1}$, KOICHIRO YAMAOKU ${ }^{1}$, AMANE KANAZAWA ${ }^{1}$, MANABU SHIOZAWA ${ }^{1}$, \\ SATOSHI KOBAYASHI ${ }^{3}$, MAKOTO UENO ${ }^{3}$, MANABU MORIMOTO ${ }^{3}$, NAOTO YAMAMOTO ${ }^{4}$, \\ TAKASHI OSHIMA ${ }^{4}$, TAKAKI YOSHIKAWA ${ }^{1}$, YASUSHI RINO ${ }^{4}$, \\ MUNETAKA MASUDA ${ }^{4}$ and SOICHIRO MORINAGA ${ }^{1}$ \\ ${ }^{1}$ Department of Gastrointestinal Surgery, Kanagawa Cancer Center; ${ }^{2}$ Molecular Pathology and Genetics Division, \\ Kanagawa Cancer Center Research Institute; ${ }^{3}$ Department of Hepatobiliary Pancreatic Oncology, Kanagawa \\ Cancer Center; ${ }^{4}$ Department of Surgery, Yokohama City University, Yokohama, Kanagawa 241-8515, Japan
}

Received April 7, 2016; Accepted March 3, 2017

DOI: $10.3892 / 01.2017 .6295$

\begin{abstract}
The predictive roles of dihydropyrimidine dehydrogenase (DPD) in patients who undergo curative resection and adjuvant chemotherapy with $\mathrm{S}-1$, which is the oral 5 -fluorouracil prodrug tegafur combined with oteracil and gimeracil, remain unclear. In the present study, the clinical data from 66 consecutive patients who underwent curative resection and received adjuvant chemotherapy with $\mathrm{S}-1$ for the treatment of pancreatic cancer at Kanagawa Cancer Center (Yokohama City, Japan) from April 2005 to March 2014 were retrospectively analyzed. The association between the DPD status and the survival and clinicopathological features were investigated. Of the 66 patients, 34 patients exhibited positive DPD expression (51.5\%). Although a significant increase in DPD expression in male patients was observed, no significant differences were identified for other clinicopathological parameters, including tumor factor or node factor, between the DPD-positive expression group and the DPD-negative expression group. The median follow-up period of the present study was 29.2 months. There was no significant difference in the 3 -year overall survival (OS) rates following surgery, which were 12.6 and $14.5 \%$ in the DPD-positive and DPD-negative expression groups, respectively $(\mathrm{P}=0.352)$. However, in a subgroup analysis, a significant difference in the 3 -year OS
\end{abstract}

Correspondence to: Dr Toru Aoyama, Department of Gastrointestinal Surgery, Kanagawa Cancer Center, 2-3-2 Nakao, Asahi-ward, Yokohama, Kanagawa 241-8515, Japan

E-mail: aoyamat@kcch.jp

*Contributed equally

Key words: pancreatic cancer, dihydropyrimidine dehydrogenase, adjuvant chemotherapy, $\mathrm{S}-1$ rates following surgery was noted, which were 58.9 and $14.5 \%$ in the DPD-high and DPD-low expression groups, respectively $(\mathrm{P}=0.019)$. The intratumoral DPD expression in curatively resected pancreatic cancer patients treated with $\mathrm{S}-1$ adjuvant chemotherapy was identified to not be useful as a predictive marker, whereas the level of DPD expression is a potential predictive marker. The results of the present study require confirmation in another cohort or in a prospective multicenter study.

\section{Introduction}

Pancreatic cancer is one of the most lethal causes of cancer-associated mortality worldwide (1). The survival outcomes of patients with pancreatic cancer have been gradually improved using effective adjuvant chemotherapies (2), and previous studies have demonstrated that the administration of gemcitabine or 5-fluorouracil (5-FU) plus folinic acid improves the progression-free and overall survival (OS) rates following surgical resection in patients with pancreatic cancer compared with surgery alone (3-7). Previously, the Japan Adjuvant Study Group of Pancreatic Cancer (JASPAC-01) trial demonstrated that $\mathrm{S}-1$, which is the oral 5-FU prodrug tegafur combined with oteracil and gimeracil (CDHP), is effective as adjuvant chemotherapy for Japanese patients undergoing curative resection for stage II disease or lower or stage III disease with combined resection of the celiac artery (8). According to these results, adjuvant chemotherapy with $\mathrm{S}-1$ is now considered to be the standard treatment following curative resection for pancreatic cancer in Japan. However, $\sim 60 \%$ of the patients still develop recurrence following curative resection followed by adjuvant S-1 therapy. To improve the clinical outcomes, it is important to identify the characteristics of patients with improved prognoses and the expression of enzymes and tumor-specific activity.

A number of enzymes serve key roles in the fluoropyrimidine metabolism. Among them, dihydropyrimidine 
dehydrogenase (DPD) is a rate-limiting enzyme in 5-FU catabolism. Increased DPD expression in tumors has been hypothesized to result in relatively low sensitivity to fluoropyrimidine-based chemotherapy (9). However, the number of published studies evaluating the clinical value of the expression of DPD in resected pancreatic cancer followed by adjuvant chemotherapy with $\mathrm{S}-1$ is limited and therefore no definitive conclusions have yet been made (10-13). In order to develop individualized adjuvant chemotherapy treatment, the characterization of genes associated with tumor sensitivity or resistance to antitumor agents using cancer tissues from the patients is required for the selection of preferable treatments.

In the present study, DPD expression was investigated in consecutive patients with pancreatic cancer who underwent curative resection followed by adjuvant chemotherapy with S-1, and the association among the DPD expression results, the clinicopathological parameters and the survival rate was evaluated.

\section{Patients and methods}

Patients. The patients were selected from the medical records of 201 consecutive patients with pancreatic cancer who underwent pancreatic surgery at Kanagawa Cancer Center (Yokohama City, Japan) from April 2005 to March 2014. The following inclusion criteria were applied: i) A pathologically diagnosed pancreatic ductal adenocarcinoma according to the definitions of the 7th edition of the International Union Against Cancer (UICC) tumor-node-metastasis (TNM) classification (14); ii) patients who initially underwent R0 or R1 resection; and iii) patients who received adjuvant chemotherapy with $\mathrm{S}-1$. The resected specimens were examined histopathologically and staged according to the 7th edition of the UICC TNM classification. Patients with other types of pancreatic cancer, including intraductal papillary mucinous neoplasm, cystadenocarcinoma or neuroendocrine tumors, or patients who underwent $\mathrm{R} 2$ resection were excluded from the present study. Of the 201 patients initially considered, 66 were eligible for inclusion in the present study. The median age was 70 years (range, 46-81 years); 36 patients were male and 30 were female. A total of 40 patients underwent pancreaticoduodenectomy, 19 underwent distal pancreatectomy (DP) and 7 underwent total pancreatectomy. The median follow-up period was 29.2 months (range, 14.6-102.8 months). The present study was approved by the Institutional Review Board Committee of the Kanagawa Cancer Center.

Surgical procedure. All pancreatic surgeries were performed in accordance with standard procedures that have been described previously (15). In cases of pancreaticoduodenectomy, lymph node dissection along the hepatoduodenal ligament, common hepatic artery, vena cava, superior mesenteric vein and the right side of the superior mesenteric artery was a standard part of the procedure. Multiple intraperitoneal drains were placed: The first was posterior to choledochojejunostomy, and the second was on the anterior surface of pancreaticojejunostomy or the closed remnant of the pancreas. In cases of DP, lymph node dissection was performed in the region of the celiac trunk and the superior mesenteric artery and vein, as well as behind the pancreas along the left side of the renal vein and the left adrenal gland. Intraperitoneal drains were placed close to the pancreatic stump.

Adjuvant chemotherapy. S-1 treatment was initiated within 10 weeks of surgery. The patients received S-1 at between 80 and $120 \mathrm{mg} / \mathrm{day}$ for 4 weeks followed by 2 weeks of rest, and treatment was continued for 6 months. The doses were modified in accordance with the JASPAC-01 trial (8); when adverse reactions appeared, the dose was decreased from 120 to $100 \mathrm{mg} /$ day or from 100 to $80 \mathrm{mg} /$ day, or administration was temporarily discontinued. Treatment was discontinued when the patient exhibited disease recurrence or adverse reactions that were uncontrollable even by dose modification or the temporary withdrawal of drug administration.

Follow-up. Patients were followed up at outpatient clinics. Hematological tests and physical examinations were performed at least every 2 weeks during adjuvant chemotherapy, and at least every 3 months for 5 years following the end of the course of adjuvant chemotherapy. The carcinoembryonic antigen and cancer antigen 19-9 tumor marker levels were evaluated at least every 3 months for 5 years. Patients underwent a computed tomography examination every 3 months during the first 3 years following surgery, and then every 6 months for 5 years following surgery. Peritoneal recurrence was defined as positive when imaging studies identified at least one of the following characteristics: Massive ascites, ascites confirmed by cytology, enhanced abdominal nodules, abnormal intestinal wall thickness, increased fat density of the intestinal mesentery, diffuse hydronephrosis or an intra-abdominal mass. When liver metastasis was suspected according to imaging studies, magnetic resonance imaging or contrast-enhanced ultrasonography was performed to confirm the diagnosis.

Immunohistochemical analysis of the DPD expression. Hematoxylin and eosin-stained slides containing specimens from each pancreatic ductal adenocarcinoma were reviewed, and a representative tumor region and the corresponding formalin-fixed paraffin-embedded tissue block was selected for use in a tissue microarray. Slides of $4-\mu \mathrm{m}$ thickness were used for immunohistochemical staining. Slides were deparaffinized in xylene for $30 \mathrm{~min}$, rehydrated using a gradient of ethanol and steamed for $5 \mathrm{~min}$. Antigen retrieval was performed with $121^{\circ} \mathrm{C}$ in a buffer with $(10 \mathrm{mM}$ sodium citrate, $\mathrm{pH}$ 6.0) in a pressure boiler. Slides remained in the pressure boiler to cool down to $90^{\circ} \mathrm{C}$ and then incubated for $20 \mathrm{~min}$ at room temperature. Endogenous peroxidase activity was blocked by immersion in $3 \%$ hydrogen peroxide at room temperature for $10 \mathrm{~min}$, with 3 TBST washes both before and after. The slices were incubated with anti-dihydropyrimidine dehydrogenase/DPYD anti rabbit polyclonal antibody (1:50; \#ABC451; EMD Millipore, Billerica, MA, USA) at room temperature for $60 \mathrm{~min}$ with 3 washes in TBST both before and after. The detection was performed according to the manufacturers protocol of Histofine Simple Stain MAX-PO Polymer (\#424141; Nichirei Bioscience, Inc., Tokyo, Japan) at room temperature for $30 \mathrm{~min}$ with 3 washes in TBST both before and after. Visualization was performed 'DAB Substrate Kit' (425011, NICHIREI BIOSCIENCES INC, Japan) used 

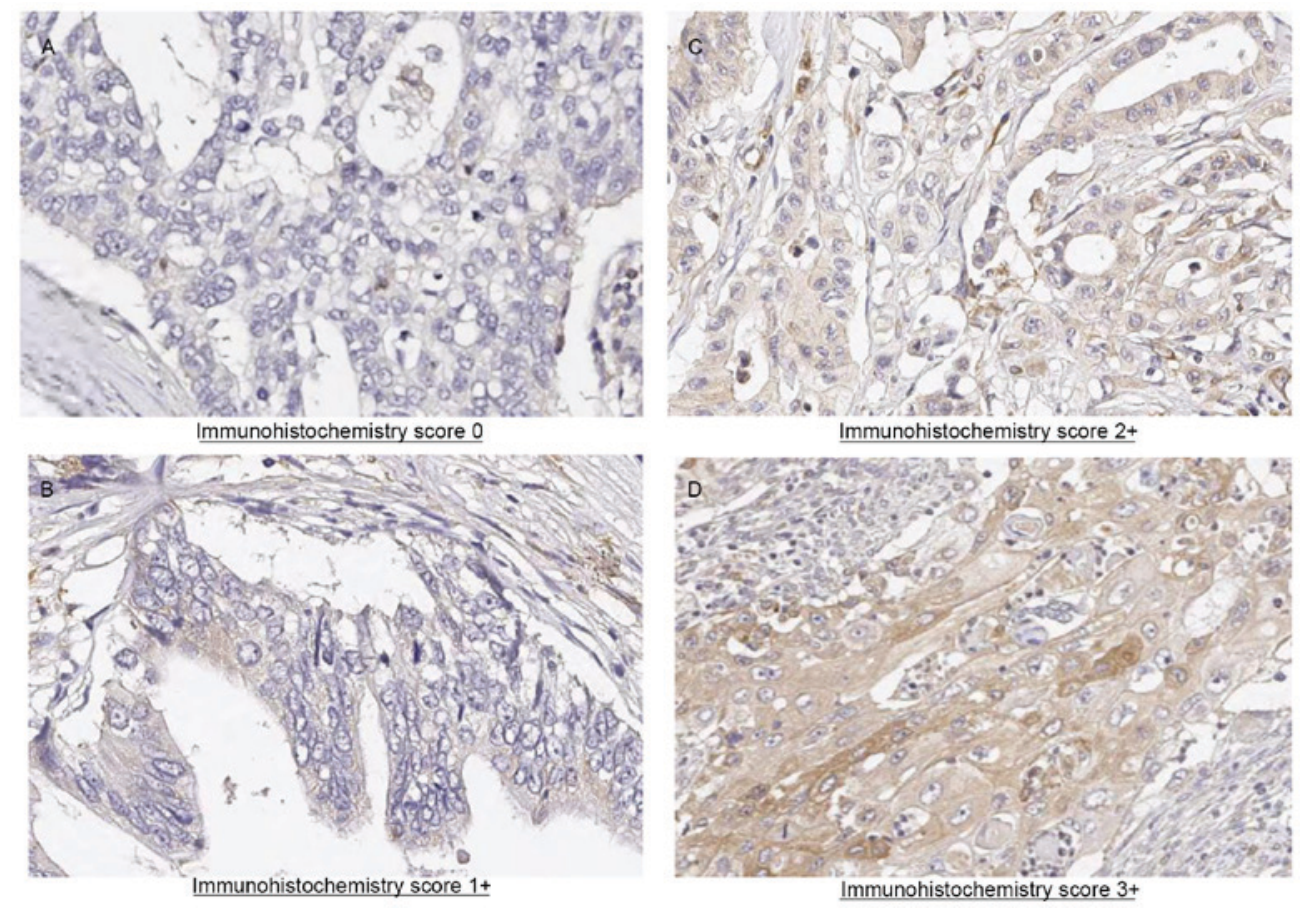

Figure 1.Immunohistochemical analysis of pancreatic cancer specimens for dihydropyrimidine dehydrogenase expression (magnification, $\mathrm{x} 200$ ). (A) Pancreatic cancer, grade 0. (B) Pancreatic cancer, grade 1. (C) Pancreatic cancer, grade 2. (D) Pancreatic cancer, grade 3 (high).

as a chromogen. Finally, slices were counterstained with hematoxylin.

The intensity of DPD staining was scored using a light microscope (CH30; Olympus Corporation, Tokyo, Japan) as follows: Grade 0, not stained; grade 1, faintly stained; grade 2, weakly stained in comparison with plasma and stroma cells; and grade 3, stained as strongly as plasma and stroma cells. For the evaluation of the cytoplasmic DPD expression, if grade 2 or 3 staining was observed in $>50 \%$ of the neoplasms, the sample was considered to exhibit positive DPD expression, whereas if grade 0 or 1 staining was observed in $>50 \%$ of tumor cells, the sample was considered to exhibit negative DPD expression. Furthermore, if grade 3 staining was observed in $>50 \%$ of the neoplasms, the sample was considered exhibit high DPD expression. The immunohistochemical evaluation of DPD expression was independently confirmed by two observers (M.M. and Y.M.) and a consensus was reached by joint review.

Evaluation and statistical analyses. The significance of the association between DPD expression and clinicopathological parameters was determined using Fisher's exact test or a $\chi^{2}$ test. The OS rate was defined as the period between surgery and mortality. The data of the patients who had not experienced an event were censored at the date of the final observation. The OS rate was evaluated using univariate and multivariate analyses. OS curves were calculated using the Kaplan-Meier estimator method and compared using the log-rank test. The univariate and multivariate survival analyses were performed using Cox's proportional hazards model. $\mathrm{P}<0.05$ was considered to indicate a statistically significant difference. The survival data were obtained from hospital records or from the city registry system. SPSS software (version 11.0J for Windows; SPSS Inc., Chicago, IL, USA) was used for all statistical analyses.

\section{Results}

Immunohistochemical analyses and the association between clinicopathological factors. Representative results of immunohistochemical staining for DPD in pancreatic adenocarcinoma tissue sections are presented in Fig. 1. Immunoreactivity was observed in the cytoplasm of cancer cells. Of the 66 tumor samples analyzed, 10 (14.5\%) exhibited negative staining, 22 samples $(31.9 \%)$ exhibited weak staining, 16 samples (23.2\%) exhibited moderate staining and 18 (26.1\%) exhibited strong staining. A total of 32 patients were assigned to the DPD-negative expression group and 34 patients were assigned to the DPD-positive expression group (Table I). In total, 9 clinicopathological factors were evaluated. Although a significant increase in DPD expression in male patients was observed, no significant difference for the other clinicopathological parameters, including $\mathrm{T}$ factor or $\mathrm{N}$ factor, was identified between the DPD-positive and DPD-negative expression groups. In an exploratory analysis according to DPD status, 18 patients were assigned to the DPD-high expression group and 48 patients were assigned to the DPD-low expression group (Table II). The clinicopathological factors were compared between the patients exhibiting high and low DPD expression. A total of 9 clinicopathological factors were evaluated. Although a significant increase in DPD expression was observed in male patients, no significant difference for the other clinicopathological parameters, including $\mathrm{T}$ factor or $\mathrm{N}$ factor, was identified between the DPD-high and DPD-low expression groups.

Survival analysis. No significant difference in the 3-year OS rates in patients exhibiting positive and negative DPD expression (12.6 and $14.5 \%$, respectively) was identified $(\mathrm{P}=0.1004$; Fig. 2). 
Table I. Comparison of the patient background between the DPD-positive and DPD-negative groups.

\begin{tabular}{|c|c|c|c|}
\hline Characteristic & $\begin{array}{l}\text { Patients exhibiting positive } \\
\text { DPD expression }(n=34)\end{array}$ & $\begin{array}{l}\text { Patients exhibiting negative } \\
\text { DPD expression }(n=32)\end{array}$ & P-value \\
\hline Median age, years (range) & $71(50-80)$ & $66(51-81)$ & 0.266 \\
\hline Sex & & & 0.025 \\
\hline Male & $23(67.6 \%)$ & $13(40.6 \%)$ & \\
\hline Female & $11(32.4 \%)$ & $19(59.4 \%)$ & \\
\hline Surgical procedure & & & 0.235 \\
\hline $\mathrm{PD}$ & $24(70.6 \%)$ & $16(50.0 \%)$ & \\
\hline DP & $8(23.5 \%)$ & $11(34.4 \%)$ & \\
\hline ТP & $2(5.9 \%)$ & $5(15.6 \%)$ & \\
\hline Median size of tumor, mm (range) & $34.5(18-105)$ & $40(15-80)$ & 0.718 \\
\hline Pathological type & & & 0.708 \\
\hline tub1 & $20(58.8 \%)$ & $19(59.4 \%)$ & \\
\hline tub2 & $10(29.4 \%)$ & $8(25.0 \%)$ & \\
\hline por & $3(8.8 \%)$ & $2(6.3 \%)$ & \\
\hline Others & $1(2.9 \%)$ & $3(9.4 \%)$ & \\
\hline Pathological T factor & & & 0.163 \\
\hline $\mathrm{T} 2$ & $1(2.9 \%)$ & $0(0.0 \%)$ & \\
\hline $\mathrm{T} 3$ & $32(94.1 \%)$ & $32(100.0 \%)$ & \\
\hline $\mathrm{T} 4$ & $1(2.9 \%)$ & $0(0.0 \%)$ & \\
\hline Pathological N factor & & & 0.351 \\
\hline N0 & $11(32.4 \%)$ & $6(18.8 \%)$ & \\
\hline N1 & $23(67.6 \%)$ & $26(81.3 \%)$ & \\
\hline Stage (7th UICC classification) & & & 0.351 \\
\hline IIA & $11(32.4)$ & $6(18.8)$ & \\
\hline IIB & $22(64.7 \%)$ & $26(81.3 \%)$ & \\
\hline III & $1(2.9 \%)$ & $0(0.0 \%)$ & \\
\hline
\end{tabular}

DPD, dihydropyrimidine dehydrogenase; PD, pancreaticoduodenectomy; DP, distal pancreatectomy; TP, total pancreatectomy; tub1, well-differentiated tubular adenocarcinoma; tub2, moderately differentiated tubular adenocarcinoma; por, poorly differentiated adenocarcinoma; T, tumor; N, node; UICC, Union for International Cancer Control.

An exploratory analysis according to the DPD status suggested that the OS time was longer in patients exhibiting high DPD expression than in patients exhibiting low DPD expression. To explore this result, a subgroup analysis was performed, with patients stratified according to high (DPD staining grade 3 ) or low (DPD staining grades 0-2) levels of DPD protein in their tumors. A significantly increased 3-year OS rate was identified in patients exhibiting high DPD expression compared with those exhibiting low DPD expression (58.9 and $14.5 \%$, respectively; $\mathrm{P}=0.0115$; Fig. 3). Furthermore, univariate and multivariate analysis demonstrated that high DPD expression was an independent risk factor for the OS rate $(\mathrm{P}=0.02$; Table III).

\section{Discussion}

In the present study, DPD status was evaluated in pancreatic adenocarcinoma patients who underwent curative resection followed by adjuvant chemotherapy with S-1, and no significant difference in the OS rate was identified between patients exhibiting positive DPD expression and patients exhibiting

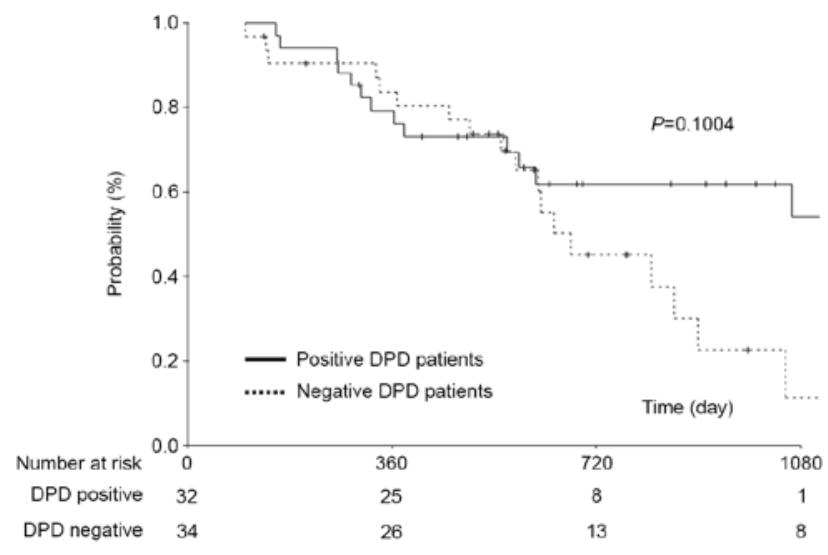

Figure 2. Comparison of overall survival between patients with pancreatic cancer exhibiting positive DPD expression with those exhibiting negative DPD expression. DPD, dihydropyrimidine dehydrogenase.

negative DPD expression. By contrast, a significant increase was identified in the OS rate of the patients exhibiting high 
Table II. Comparison of the patient background between the DPD-high and DPD-low expression groups.

\begin{tabular}{|c|c|c|c|}
\hline Characteristic & $\begin{array}{l}\text { Patients exhibiting high } \\
\text { DPD expression }(n=18)\end{array}$ & $\begin{array}{l}\text { Patients exhibiting low } \\
\text { DPD expression }(n=48)\end{array}$ & P-value \\
\hline Median age, years (range) & $71.5(56-79)$ & $68.5(46-81)$ & 0.527 \\
\hline Sex & & & 0.001 \\
\hline Male & $16(88.9 \%)$ & $20(41.7 \%)$ & \\
\hline Female & $2(11.1 \%)$ & $28(58.3 \%)$ & \\
\hline Surgical procedure & & & 0.686 \\
\hline $\mathrm{PD}$ & $12(66.7 \%)$ & $28(58.3 \%)$ & \\
\hline $\mathrm{DP}$ & $5(27.8 \%)$ & $14(29.2 \%)$ & \\
\hline $\mathrm{TP}$ & $1(5.6 \%)$ & $6(12.5 \%)$ & \\
\hline Median size of tumor, mm (range) & $33.5(15-90)$ & $38(15-105)$ & 0.319 \\
\hline Pathological type & & & 0.323 \\
\hline tub1 & $9(50.0 \%)$ & $30(62.5 \%)$ & \\
\hline tub2 & $7(38.9 \%)$ & $11(22.9 \%)$ & \\
\hline por & $2(11.1 \%)$ & $3(6.3 \%)$ & \\
\hline Others & $0(0.0 \%)$ & $4(8.3 \%)$ & \\
\hline Pathological T factor & & & 0.727 \\
\hline $\mathrm{T} 3$ & $18(100.0 \%)$ & $47(97.9 \%)$ & \\
\hline $\mathrm{T} 4$ & $0(0.0 \%)$ & $1(2.1 \%)$ & \\
\hline Pathological $\mathrm{N}$ factor & & & 0.038 \\
\hline No & $8(44.4 \%)$ & $9(18.8 \%)$ & \\
\hline N1 & $10(55.6 \%)$ & $39(81.3 \%)$ & \\
\hline Stage (7th UICC classification) & & & 0.101 \\
\hline IIA & $8(44.4 \%)$ & $9(18.8 \%)$ & \\
\hline IIB & $10(55.6 \%)$ & $38(79.2 \%)$ & \\
\hline III & $0(0.0 \%)$ & $1(2.1 \%)$ & \\
\hline
\end{tabular}

DPD, dihydropyrimidine dehydrogenase; PD, pancreaticoduodenectomy; DP, distal pancreatectomy; TP, total pancreatectomy; tub1, well-differentiated tubular adenocarcinoma; tub2, moderately differentiated tubular adenocarcinoma; por, poorly differentiated adenocarcinoma; T, tumor; N, node; UICC, Union for International Cancer Control.

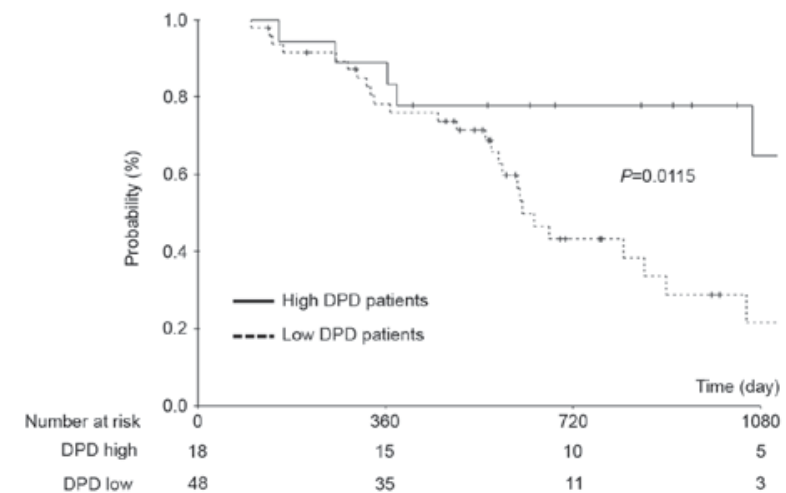

Figure 3. Comparison of overall survival between patients with pancreatic cancer exhibiting high DPD expression with those exhibiting low DPD expression. DPD, dihydropyrimidine dehydrogenase.

DPD expression compared with patients exhibiting low DPD expression. These results suggested that DPD status is a potential predictive marker in patients with pancreatic cancer who undergo curative resection with S-1 adjuvant chemotherapy.
Previous studies have investigated the presence and effect of DPD protein overexpression or gene amplification in patients. These studies have identified that DPD expression is increased in between 30 and $50 \%$ of patients. Kondo et al (16) evaluated the intratumoral DPD expression in pancreatic carcinoma using immunohistochemical methods in 86 Japanese patients with pancreatic carcinoma who were treated with adjuvant S-1-based chemotherapy. This study identified that high DPD expression was observed in 35 (41\%) patients. In addition, Shimoda et al (13) determined DPD expression in 57 Japanese patients with pancreatic carcinoma who were treated with adjuvant S-1 or gemcitabine chemotherapy. This study identified that high DPD expression was observed in 52\% of the patients in the S-1 group. These results were similar to those of the present study. Therefore, the incidence of high DPD expression may be between 40 and $50 \%$ in patients with resectable pancreatic cancer.

With regard to the association between the DPD expression and clinicopathological factors, a study by Kondo et al (11) identified that there were no significant differences in clinicopathological factors, including UICC pathological $\mathrm{T}$ factor and lymph node status, between the patients exhibiting high 
Table III. Univariate and multivariate analyses of risk factors for the overall survival rates of patients exhibiting high DPD expression compared with those exhibiting low DPD expression.

\begin{tabular}{|c|c|c|c|c|c|c|c|}
\hline \multirow[b]{2}{*}{ Factor } & \multirow[b]{2}{*}{$\mathrm{n}$} & \multicolumn{3}{|c|}{ Univariate analysis } & \multicolumn{3}{|c|}{ Multivariate analysis } \\
\hline & & OR & $95 \%$ CI & P-value & OR & $95 \%$ CI & P-value \\
\hline Sex & & & & 0.662 & & & \\
\hline Female & 30 & 1 & & & & & \\
\hline Male & 36 & 1.177 & $0.567-2.441$ & & & & \\
\hline Age, years & & & & 0.532 & & & \\
\hline$<70$ & 29 & 1 & & & & & \\
\hline$\geq 70$ & 37 & 1.259 & $0.612-2.587$ & & & & \\
\hline Size of tumor, mm & & & & 0.352 & & & \\
\hline$<38$ & 33 & 1 & & & & & \\
\hline$\geq 38$ & 33 & 1.42 & $0.678-2.974$ & & & & \\
\hline Tumor location & & & & 0.239 & & & \\
\hline Body or tail & 19 & 1 & & & & & \\
\hline Head & 47 & 1.709 & $0.700-4.169$ & & & & \\
\hline Pathological $\mathrm{N}$ factor & & & & 0.034 & & & \\
\hline N0 & 17 & 1 & & & & & \\
\hline N1 & 49 & 3.627 & $1.099-11.971$ & & & & \\
\hline DPD status & & & & 0.023 & & & 0.02 \\
\hline Low & 48 & 1 & & & 1 & & \\
\hline High & 18 & 3.078 & $1.164-8.135$ & & 3.183 & $1.197-8.467$ & \\
\hline
\end{tabular}

DPD, dihydropyrimidine dehydrogenase; OR, odds ratio; CI, confidence interval; N, node.

and low DPD expression. A study by Nakahara et al (17) also identified similar results in 18 patients. In the present study, no significant differences in the clinicopathological factors between the patients exhibiting high and low DPD expression were identified. Therefore, DPD expression appears to be independent of clinicopathological factors.

In the present study, DPD expression was not identified to be a predictive marker in patients with pancreatic cancer who underwent curative resection with S-1 adjuvant chemotherapy. However, DPD status is a potential predictive marker in patients with pancreatic cancer who undergo curative resection with S-1 adjuvant chemotherapy. Indeed, multivariate OS analysis in the study by Kondo et al (16) identified that high DPD expression is one of the predictive markers (hazard ratio, 1.98; $95 \%$ confidence interval, $1.06-3.71 ; \mathrm{P}=0.03)$.

It is hypothesized that tumor cells which exhibit high DPD expression are resistant to fluoropyrimidine-based therapy (18). In the present study, survival analysis results indicated that the antitumor effect of S-1 for pancreatic cancer was not influenced by intratumoral DPD gene expression; furthermore, improved survival was observed in patients exhibiting high DPD expression. There are potential explanations for these conflicting results. First, similar results were observed in previous studies. In a study by Sasako et al (19) an association between thymidylate synthase (TS), which is the rate-limiting enzyme in the de novo synthesis of 2'-deoxy-thymidine-5'-monophosphate, and DPD expression in tumors and the clinical outcomes in stage II/III gastric cancer was identified. This study identified that high TS and DPD gene expression in tumors was associated with an enhanced benefit from postoperative adjuvant S-1 treatment in gastric cancer. This study demonstrated that S-1 caused certain effects not exerted by other fluoropyrimidines. Ichikawa et al (20) described "some effect" that was explained by the inhibition of intratumoral DPD by CDHP, which is contained in S-1 therapy. Secondly, the intratumoral DPD mRNA expression level in pancreatic cancer was significantly increased compared with in colorectal cancer and gastric cancer (21). In addition, Takechi et al (22) demonstrated that the in vitro antitumor activity of 5-FU against tumor cells with low DPD expression levels was not appreciably affected by the addition of CDHP (22). Therefore, in pancreatic cancer, it is considered that high DPD expression influences S-1 treatment and this discrepancy is dependent on the difference between S-1 and other 5-FU agents.

Careful attention is required when interpreting the results of the present study owing to a number of potential limitations. First, the present study was a retrospective analysis which was performed at a single institution. There is the possibility that the results were incidental. Secondly, there was a subjective bias in the immunohistochemical evaluation of the DPD expression. The methods of evaluating DPD expression were not standardized. Furthermore, the appropriate DPD threshold value is unclear. Considering these limitations, the results of the present study require confirmation in another cohort or in a prospective multicenter study. 
DPD status is considered to be a potentially useful predictive marker in pancreatic cancer patients who undergo curative resection with S-1 adjuvant chemotherapy. However, the results of the present study require confirmation in another cohort or in a prospective multicenter study.

\section{Acknowledgements}

The present study was supported by the Kanagawa Prefectural Hospitals Cancer Fund.

\section{References}

1. Siegel R, Naishadham D and Jemal A: Cancer statistics, 2012. CA Cancer J Clin 62: 10-29, 2012.

2. Vincent A, Herman J, Schulick R, Hruban RH and Goggins M: Pancreatic cancer. Lancet 378: 607-620, 2011.

3. Burris HA III, Moore MJ, Andersen J, Green MR, Rothenberg ML, Modiano MR, Cripps MC, Portenoy RK, Storniolo AM, Tarassoff $\mathrm{P}$, et al: Improvements in survival and clinical benefit with gemcitabine as first-line therapy for patients with advanced pancreas cancer: A randomized trial. J Clin Oncol 15: 2403-2413, 1997.

4. Neoptolemos JP, Stocken DD, Friess H, Bassi C, Dunn JA, Hickey $\mathrm{H}$, Beger $\mathrm{H}$, Fernandez-Cruz L, Dervenis C, Lacaine $\mathrm{F}$, et al: A randomized trial of chemoradiotherapy and chemotherapy after resection of pancreatic cancer. N Engl J Med 350: 1200-1210, 2004

5. Oettle H, Post S, Neuhaus P, Gellert K, Langrehr J, Ridwelski K, Schramm H, Fahlke J, Zuelke C, Burkart C, et al: Adjuvant chemotherapy with gemcitabine vs observation in patients undergoing curative-intent resection of pancreatic cancer: A randomized controlled trial. JAMA 297: 267-277, 2007.

6. Neoptolemos JP, Stocken DD, Tudur Smith C, Bassi C, Ghaneh P, Owen E, Moore M, Padbury R, Doi R, Smith D and Büchler MW: Adjuvant 5-fluorouracil and folinic acid vs observation for pancreatic cancer: Composite data from the ESPAC-1 and -3(v1) trials. Br J Cancer 100: 246-250, 2009.

7. Neoptolemos JP, Stocken DD, Bassi C, Ghaneh P, Cunningham D, Goldstein D, Padbury R, Moore MJ, Gallinger S, Mariette C, et al: Adjuvant chemotherapy with fluorouracil plus folinic acid vs gemcitabine following pancreatic cancer resection: A randomized controlled trial. JAMA 304: 1073-1081, 2010.

8. Maeda A, Boku N, Fukutomi A, Kondo S, Kinoshita T, Nagino M and Uesaka K: Randomized phase III trial of adjuvant chemotherapy with gemcitabine versus S-1 in patients with resected pancreatic cancer: Japan Adjuvant Study Group of Pancreatic Cancer (JASPAC-01). Jpn J Clin Oncol 38: 227-229, 2008.

9. Salonga D, Danenberg KD, Johnson M, Metzger R, Groshen S, Tsao-Wei DD, Lenz HJ, Leichman CG, Leichman L, Diasio RB and Danenberg PV: Colorectal tumors responding to 5-fluorouracil have low gene expression levels of dihydropyrimidine dehydrogenase, thymidylate synthase, and thymidine phosphorylase. Clin Cancer Res 6: 1322-1327, 2000.

10. Saif MW, Hashmi S, Bell D and Diasio RB: Prognostication of pancreatic adenocarcinoma by expression of thymidine phosphorylase/dihydropyrimidine dehydrogenase ratio and its correlation with survival. Expert Opin Drug Saf 8: 507-514, 2009.
11. Kondo N, Murakami Y, Uemura K, Sudo T, Hashimoto Y, Nakashima A, Ohge $\mathrm{H}$ and Sueda T: Prognostic impact of dihydropyrimidine dehydrogenase expression on pancreatic adenocarcinoma patients treated with S-1-based adjuvant chemotherapy after surgical resection. J Surg Oncol 104: 146-154, 2011.

12. Nakamura A, Hayashi K, Nakajima G, Kamikozuru H, Okuyama R, Kuramochi H, Hatori T and Yamamoto M: Impact of dihydropyrimidine dehydrogenase and $\gamma$-glutamyl hydrolase on the outcomes of patients treated with gemcitabine or S-1 as adjuvant chemotherapy for advanced pancreatic cancer. Exp Ther Med 2: 1097-1103, 2011.

13. Shimoda M, Kubota K, Shimizu T and Katoh M: Randomized clinical trial of adjuvant chemotherapy with S-1 versus gemcitabine after pancreatic cancer resection. Br J Surg 102: 746-754, 2015.

14. Scoazec JY and Sabourin JC: 2010: The seventh edition of the TNM classification. Ann Pathol 30: 2-6, 2010 (In French).

15. Aoyama T, Murakawa M, Katayama Y, Yamaoku K, Kanazawa A, Higuchi A, Shiozawa M, Morimoto M, Yoshikawa T, Yamamoto $\mathrm{N}$, et al: Impact of postoperative complications on survival and recurrence in pancreatic cancer. Anticancer Res 35: 2401-2409, 2015

16. Kondo N, Murakami Y, Uemura K, Sudo T, Hashimoto Y, Nakashima A and Sueda T: Combined analysis of dihydropyrimidine dehydrogenase and human equilibrative nucleoside transporter 1 expression predicts survival of pancreatic carcinoma patients treated with adjuvant gemcitabine plus S-1 chemotherapy after surgical resection. Ann Surg Oncol 19 (Suppl 3): S646-S655, 2012

17. Nakahara O, Takamori H, Tanaka H, Sakamoto Y, Ikuta Y, Furuhashi S, Watanabe M, Beppu T, Hirota M, Kanemitsu K and Baba H: Clinical significance of dihydropyrimidine dehydrogenase and thymidylate synthase expression in patients with pancreatic cancer. Int J Clin Oncol 15: 39-45, 2010.

18. Wang WB, Yang Y, Zhao YP, Zhang TP, Liao Q and Shu H: Recent studies of 5-fluorouracil resistance in pancreatic cancer. World J Gastroenterol 20: 15682-15690, 2014.

19. Sasako M, Terashima M, Ichikawa W, Ochiai A, Kitada K, Kurahashi I, Sakuramoto S, Katai H, Sano T and Imamura H: Impact of the expression of thymidylate synthase and dihydropyrimidine dehydrogenase genes on survival in stage II/III gastric cancer. Gastric Cancer 18: 538-548, 2015.

20. Ichikawa W, Takahashi T, Suto K, Yamashita T, Nihei Z, Shirota Y, Shimizu M, Sasaki Y and Hirayama R: Thymidylate synthase predictive power is overcome by irinotecan combination therapy with S-1 for gastric cancer. Br J Cancer 91: 1245-1250, 2004.

21. Kuramochi H, Hayashi K, Uchida K, Nakajima G, Hatori T, Danenberg KD, Danenberg PV and Yamamoto M: High intratumoral dihydropyrimidine dehydrogenase mRNA levels in pancreatic cancer associated with a high rate of response to S-1. Cancer Chemother Pharmacol 63: 85-89, 2008.

22. Takechi T, Fujioka A, Matsushima E and Fukushima M: Enhancement of the antitumour activity of 5-fluorouracil (5-FU) by inhibiting dihydropyrimidine dehydrogenase activity (DPD) using 5-chloro-2,4-dihydroxypyridine (CDHP) in human tumour cells. Eur J Cancer 38: 1271-1277, 2002. 